Article

\title{
High-Performance Low-Pass Filter Using Stepped Impedance Resonator and Defected Ground Structure
}

\author{
Jin Zhang ${ }^{1,2,3, * \mathbb{C}}$, Ruosong Yang ${ }^{2}$ and Chen Zhang ${ }^{2}$ \\ 1 School of Electronic and Information Engineering, Jinling Institute of Technology, Nanjing 211169, China \\ 2 National and Local Joint Engineering Laboratory of RF Integration and Micro-Assembly Technology, \\ Nanjing 210023, China; 1018020806@njupt.edu.cn (R.Y.); 1018020802@njupt.edu.cn (C.Z.) \\ 3 School of Electrical Engineering, Southeast University, Nanjing 210096, China \\ * Correspondence: jinzhang@jit.edu.cn; Tel.: +86-298-618-8701
}

Received: 26 January 2019; Accepted: 1 April 2019; Published: 4 April 2019

\begin{abstract}
A microstrip low-pass filter (LPF) using reformative stepped impedance resonator (SIR) and defected ground structure (DGS) is proposed in this paper. The proposed filter not only possesses the advantage of high frequency selectivity of SIR hairpin LPF with internal coupling, but also possesses the large stop-band (SB) bandwidth by adjusting the number and area of DGS units. The LPF proposed in this paper possesses the properties of miniaturization, wide SB, high selectivity, and low pass-band ripple (PBR) simultaneously. The characteristic parameters of the proposed LPF is that: the pass-band (PB) is $0 \sim 2 \mathrm{GHz}$, the PBR is $0.5 \mathrm{~dB}$, the $\mathrm{SB}$ range is from $2.4 \mathrm{GHz}$ to $9 \mathrm{GHz}$ when the attenuation is under $20 \mathrm{~dB}$, and the maximal attenuation could reach $45 \mathrm{~dB}$ in the SB. The size of this proposed LPF is $0.13 \lambda \times 0.09 \lambda ; \lambda$ is the corresponding wavelength of the upper PB edge frequency of $2 \mathrm{GHz}$.
\end{abstract}

Keywords: low-pass filter (LPF); stepped impedance resonator (SIR); hairpin resonator; internal coupling; defected ground structure (DGS)

\section{Introduction}

In recent years, the miniaturized microstrip low-pass filter (LPF) with high frequency selectivity and wide stop-band (SB) is widely used in a RF/microwave circuit such as satellite and mobile communication systems. However, based on step impedance resonator (SIR), the traditional microstrip LPFs implemented by Richard transform and Kuroda rule can only constitute the LPFs of the type of Butterworth and Chebyshev responses [1]. The responses of these two filters with single-sections exhibit poor frequency selectivity. Therefore, in order to obtain a steep selecting edge, the number of filter sections must be increased [2,3]. At the same time, the insertion loss in the pass-band (PB) and the physical size of the filter will be increased. On the basis of a previous study [4], Reference [5] proposed a PCB LPF using a semi-lumped parallel resonance circuit which exhibits an elliptic function response. In Reference [6], the coupling between two LP hairpin filters was performed to obtain a bandpass response. These kinds of filters achieve high frequency selectivity, but the SB width is not wide enough, and the attenuation out of the band is insufficient.

In order to realize a microstrip LPF with the characteristics of miniaturization, high selectivity and wide SB suppression at the same time, the research hotspot of the defected ground structure (DGS) of nearly two decades is combined with the SIR hairpin filter in this paper. The DGS was proposed to design LPF by Korean scholars D. Ahn et al. in 2001 [7]. From then on, more and more research findings have used DGS to design a high performance microstrip filter. Nechel et al. [8] introduced metamodels in filter design by use of a DGS. The optimal time of the design process decreases drastically, however, the simple slot DGS analyzed in the reference is not suitable for filter design for high frequency 
selectivity. In Reference [9], asymmetrical Pi-shaped DGSs with Koch fractal curve were used to design compact LPF, however, the multiple asymmetrical fractal structures undoubtedly increase the filter's size. Zeng et al. [10] used compact DGSs with mutual capacity and inductive coupling to broaden the bandwidth and the rejection ratio in SB range; the shortcoming of this tight coupling design is that it increases the PB insertion loss. A compact LPF was designed by combining several fractal DGSs with a conventional multiple stub filter in Reference [11]. However, the size of the filter designed in the reference appears insufficiently compact due to the usage of conventional multiple stub LPF in the top layer.

In this paper, we use the SIR hairpin resonator with internal coupling to make the filter more compact and use the properties of the DGS to widen the SB by optimization design. This paper is organized as follows. In Section 2, the electrical characteristics of one-, two- and three-DGS are investigated. A compact LPF with high frequency selectivity is proposed based on SIR hairpin with internal coupling in Section 3. A miniaturization, high selectivity and wide SB suppression microstrip LPF are simulated and experimentally validated in Section 4 . Finally, Section 5 gives some concluding remarks.

\section{The Electrical Properties of DGS}

The DGS etched on the grounding metal plate for the microstrip circuit can be square-headed, semi-circular-headed dumbbell-shaped, and snake-shaped patterns. In this paper, the square-headed dumbbell-shaped DGS is applied. The 3-D model of one-, two- and three-DGS structures are shown in Figure 1a-c.

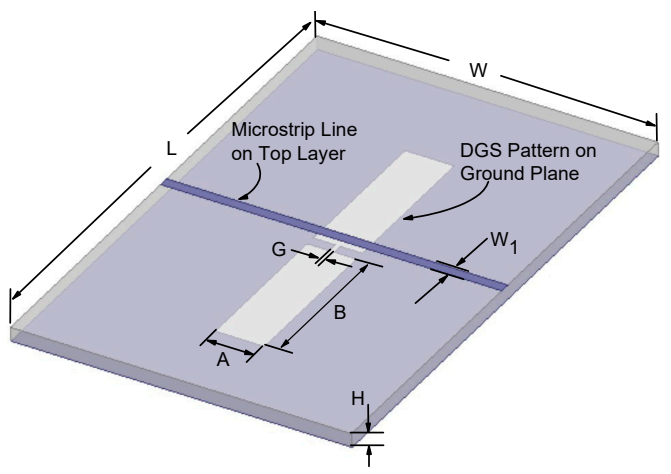

(a)

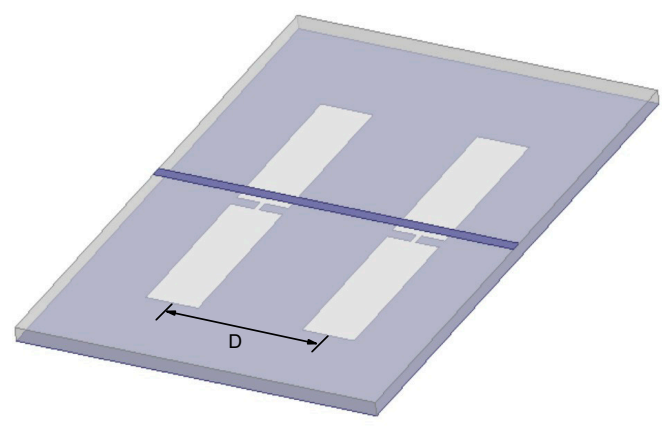

(b)

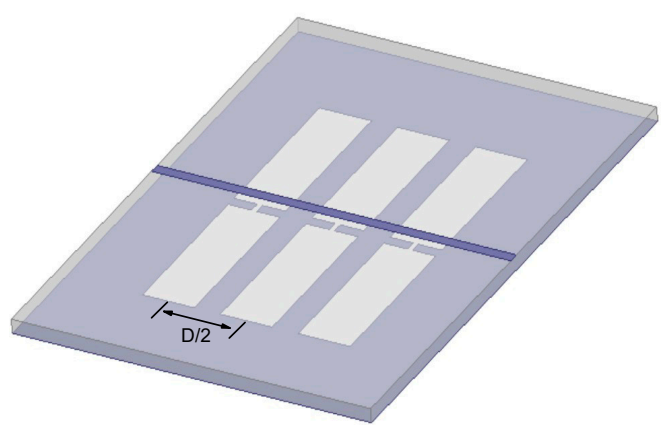

(c)

Figure 1. 3-D model of (a) one-, (b) two- and (c) three-DGS structures.

The model of one-DGS structure in Figure 1a is simulated in Ansoft HFSS. This model is designed on PTPE composite medium copper-clad foil plate with a relative dielectric constant $\varepsilon_{\mathrm{r}}=10.8$ and a loss tangent of 0.0035 . The distance of the middle slots is represented by $G$, the linewidth of microstrip line by $W_{1}$ for $50-\Omega$ characteristic impedance, and the width of DGS by $A$. The size of the whole model is about $L \times W \times H$. The detailed numerical values of the model are listed in Table 1. Figure 2a shows 
the simulated frequency responses of the return loss $S_{11}$ and the insertion loss $S_{21}$ of one-DGS case when the length $B$ of DGS are $5.8,6.8,7.8$, and $8.3 \mathrm{~mm}$, respectively.

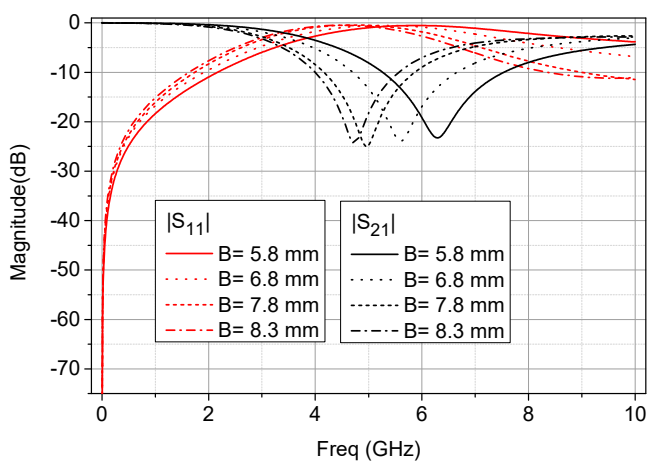

(a)

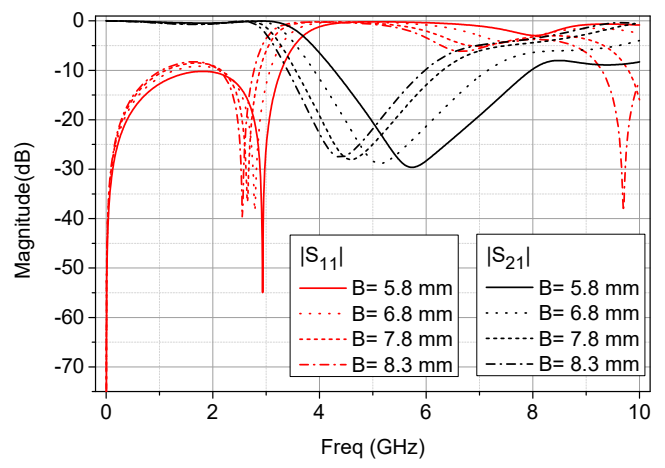

(b)

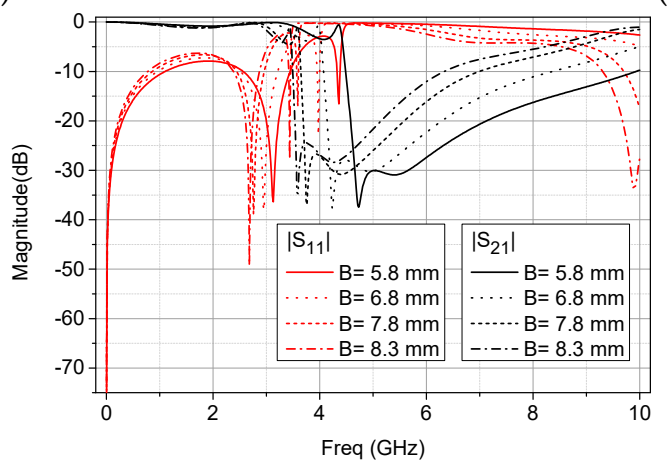

(c)

Figure 2. Frequency responses of return loss and insertion loss of the model of (a) one-DGS, (b) two-DGS and (c) three-DGS.

Table 1. Filter specifications.

\begin{tabular}{cccc}
\hline Dielectric Substrate & Values & Model & Values (mm) \\
\hline$\varepsilon_{\mathrm{r}}$ & 10.8 & $L$ & 20 \\
Loss tangent & 0.0035 & $W$ & 13.8 \\
& & $H$ & 0.5 \\
\hline SIR hairpin filter & Values (mm) & DGS & Values (mm) \\
\hline$W_{1}, W_{2}, W_{3}$ & $0.396,0.232$, & $G$ & 0.2 \\
$L_{1}$ & 0.9 & $A .92$ & \\
$L_{2}, L_{3}, L_{4}, L_{5}$ & $1,2.626,0.796$, & $D$ & 2 \\
$S$ & 6.3 & $B_{1}, B_{2}$ & $7.8,8.3$ \\
\hline
\end{tabular}

The transmission zero of the one-DGS structure shifts to the lower frequency band with the increasing length of $\mathrm{B}$ as shown in Figure 2a. The frequencies of the transmission zero are 6.28 GHz, $5.62 \mathrm{GHz}, 4.96 \mathrm{GHz}$, and $4.69 \mathrm{GHz}$, respectively. Further study shows that the frequencies of transmission zeros decrease with the increasing areas of DGS. Actually, the electrical property of the model of one DGS under the microstrip is equivalent to that of a parallel L-C resonator circuit. The growing area of the DGS corresponds to increasing the equivalent inductance of the parallel resonance circuit; the transmission zero therefore moves to the low frequency with increasing area of the DGS.

Similarly, the simulation frequency response characters of the models consisted of twoand three-DGS units which are presented in Figure 1b,c and Figure 2b,c, respectively. The distance 
between adjacent DGSs for two- and three-DGS models are D/2 $=3 \mathrm{~mm}$. The sizes of the other parts are set up identical to the one-DGS case.

The transmission zeros of two- and three-DGS structures also move to the low frequency band with the increasing length of $B$ as shown in Figure $2 b, c$. By comparing the Figure $2 a-c$, the frequency positions of transmission zeros decrease with the increasing number of DGS, and the width of the transition band (TB) between PB and SB greatly reduced in the three-DGS case. The interpretation of this phenomenon can be illustrated in Figure 3.

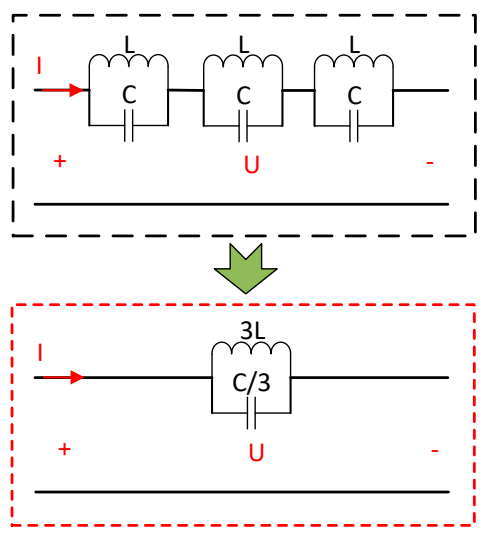

Figure 3. The equivalent circuits of the three-DGS model in Figure 2c.

The equivalent circuit model of the three-DGS model can be modeled by three parallel L-C resonators, as shown in the black dashed box in Figure 3. The mutual coupling effects between each resonator are ignored because of a greater distance between the adjacent DGSs. The relations of the electric parameters for this circuit can be expressed as

$$
U=I\left(3 \cdot \frac{1}{\frac{1}{j \omega L}+j \omega C}\right)
$$

Via simple mathematical transformation, Equation (1) converts into Equation (2)

$$
U=I\left[\frac{1}{\frac{1}{j \omega(3 L)}+j \omega(C / 3)}\right]
$$

The single equivalent parallel L-C resonator in the red dotted line in Figure 3 reflects the relations of the electric parameters in Equation (2). Because the area of the DGS mainly affects the equivalent inductance of the parallel resonator, the three lengthening side lengths of $B$ triple the parallel inductance. From DGS simulation in Figure 2, it is known that the structure of DGS has the characteristics of low-pass filtering. Its low-pass characteristics can be adjusted by changing the defected area and shape etched on the grounding plate. This characteristic is applied to broaden the SB width of the SIR hairpin LPF in Section 3.

\section{Investigation of Proposed SIR Hairpin LPF}

To realize the function of filtering, a filter requires one or more units of resonant structure. The resonant elements of SIR structure for filter designing is an effective way to realize compact filters. Lung-Hwa Hsieh et al. proposed a LPF using a SIR hairpin unit [2]. On this basis, combined with M. Makimoto's monograph [12], the following LPF with high attenuation edges are proposed. The cell structure is shown in Figure 4.

The equivalent circuit of the resonator shown in Figure 4 can be regarded as a parallel connection of a single transmission line and two parallel lines with internal coupling. The characteristic impedance 
and electric length of the single transmission line are $Z_{S}$ and $\theta_{T}$, respectively. The characteristic impedance (the electric length) of the even and odd modes of the parallel lines with internal coupling are $Z_{p e}, Z_{p o}\left(\theta_{p e}, \theta_{p o}\right)$, respectively. The $A$ matrix of the single transmission line and the parallel coupling line are expressed as $A_{I}$ and $A_{I I}$, respectively.

$$
\begin{gathered}
A_{I}=\left[\begin{array}{ll}
A_{1} & B_{1} \\
C_{1} & D_{1}
\end{array}\right] \\
=\left[\begin{array}{cc}
\cos \theta_{T} & j Z_{S} \sin \theta_{T} \\
\frac{j \sin \theta_{T}}{Z_{S}} & \cos \theta_{T}
\end{array}\right] \\
A_{I I}=\left[\begin{array}{ll}
A_{2} & B_{2} \\
C_{2} & D_{2}
\end{array}\right]
\end{gathered}
$$

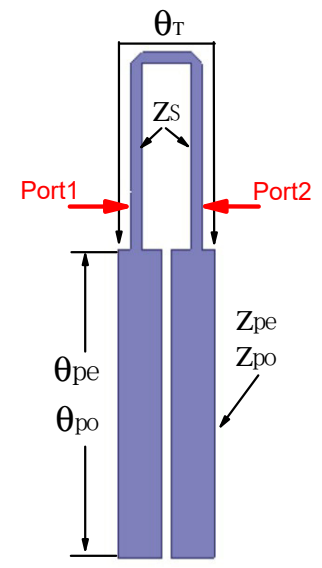

Figure 4. SIR hairpin with internal coupling.

$$
=\left[\begin{array}{cc}
\frac{Z_{p e} \cot \theta_{p e}+Z_{p o} \cot \theta_{p o}}{Z_{p e} \cot \theta_{p e}-Z_{p o} \cot \theta_{p o}} & -j \frac{2 Z_{p e} Z_{p o} \cot \theta_{p e} \cot \theta_{p o}}{Z_{p e} \cot \theta_{p e}-Z_{p o} \cot \theta_{p o}} \\
\frac{j 2}{Z_{p e} \cot \theta_{p e}-Z_{p o} \cot \theta_{p o}} & \frac{Z_{p e} \cot \theta_{p e}+Z_{p o} \cot \theta_{p o}}{Z_{p e} \cot \theta_{p e}-Z_{p o} \cot \theta_{p o}}
\end{array}\right]
$$

The matrix $A_{T}$ is defined as the total $A$ matrix of the above two parallel circuits.

$$
A_{T}=\left[\begin{array}{cc}
A_{t} & B_{t} \\
C_{t} & D_{t}
\end{array}\right]
$$

where

$$
\begin{gathered}
A_{t}=\frac{A_{1} B_{2}+A_{2} B_{1}}{B_{1}+B_{2}} \\
B_{t}=\frac{B_{1} B_{2}}{B_{1}+B_{2}} \\
C_{t}=\frac{-\left(A_{2}-A_{1}\right)\left(D_{2}-D_{1}\right)-\left(B_{2}+B_{1}\right)\left(C_{2}+C_{1}\right)}{B_{1}+B_{2}} \\
D_{t}=\frac{D_{1} B_{2}+D_{2} B_{1}}{B_{1}+B_{2}}
\end{gathered}
$$


where $D_{t}=A_{t}$ due to $D_{1}=A_{1}$ and $D_{2}=A_{2}$. A load impedance $Z_{L}$ is connected to the terminal in the circuit. The input admittance $Y_{I}$ can be calculated based on Equation (5):

$$
Y_{I}=\frac{C_{t}+\frac{D_{t}}{Z_{L}}}{A_{t}+\frac{B_{t}}{Z_{L}}}
$$

The resonance condition is met under $Y_{I}=0$ and $Z_{L}=\infty$, therefore, substituting the calculated value in Equation (5), the resonance condition is obtained as follows:

$$
\begin{gathered}
\left(Z_{p e} \cdot Z_{p o} \cot \theta_{p e} \cot \theta_{p o}-Z_{S}^{2}\right) \sin \theta_{T}+Z_{S}\left(Z_{p e} \cot \theta_{p e}+Z_{p o} \cot \theta_{p o}\right) \cos \theta_{T} \\
-Z_{S}\left(Z_{p e} \cot \theta_{p e}-Z_{p o} \cot \theta_{p o}\right)=0
\end{gathered}
$$

Based on the resonance condition of Equation (8), the characteristics of the above resonator can be analyzed using the microwave simulator method [12]. Figure 5 shows the geometry of the resonator connected with two tapped microstrip lines and its equivalent circuit diagram.

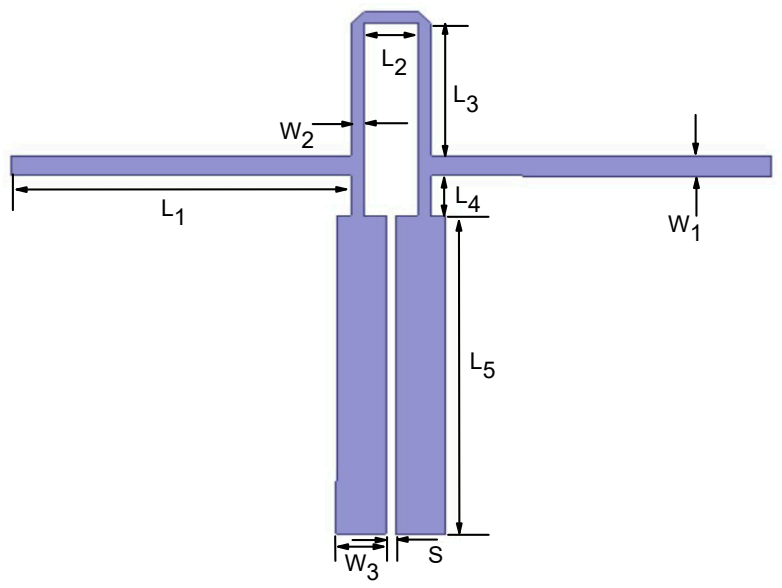

(a)

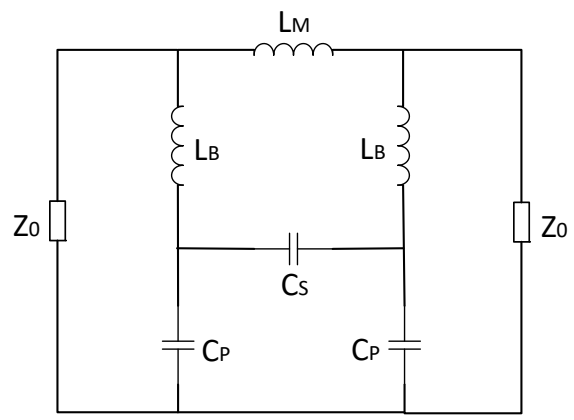

(b)

Figure 5. (a) Layout of LPF constructed by SIR hairpin with internal coupling; (b) equivalent circuit diagram of microstrip LPF.

The nonideal inductance $L_{M}$ in Figure $5 \mathrm{~b}$ is the equivalent inductance of $L_{3}, L_{2}, L_{3}$ and two $45^{\circ}$ bends in Figure $5 a . L_{B}$ is the equivalent inductance of $L_{4}$. The non-ideal capacitances $C_{S}, C_{P}$ are equivalent to the capacitances generated by two open coupling lines, in which $C_{S}$ is generated by the coupling effect of two lines; $C_{P}$ is generated by the coupling between the terminal microstrip line and the ground plate; and $Z_{0}$ is the characteristic impedance of two feeding lines. The equivalent circuit in Figure $5 \mathrm{~b}$ shows that the proposed structure has the property of an elliptic function-like LPF. The corresponding circuit model in Figure $5 \mathrm{a}$ is simulated by ADS software. The simulation is to design a LPF with a cut-off frequency of $2 \mathrm{GHz}$ under the condition that the relative dielectric constant of the microstrip substrate is 10.8 and the thickness of the substrate is $0.5 \mathrm{~mm}$. The simulated schematic in ADS software is shown in Figure 6a. To improve the frequency selectivity, the structural dimensions of the hairpin in Figure 5a are obtained through software optimizing measures, which are listed in Table 1. The transmission characteristics of the filters tuned by software are shown in Figure $6 \mathrm{~b}$. 


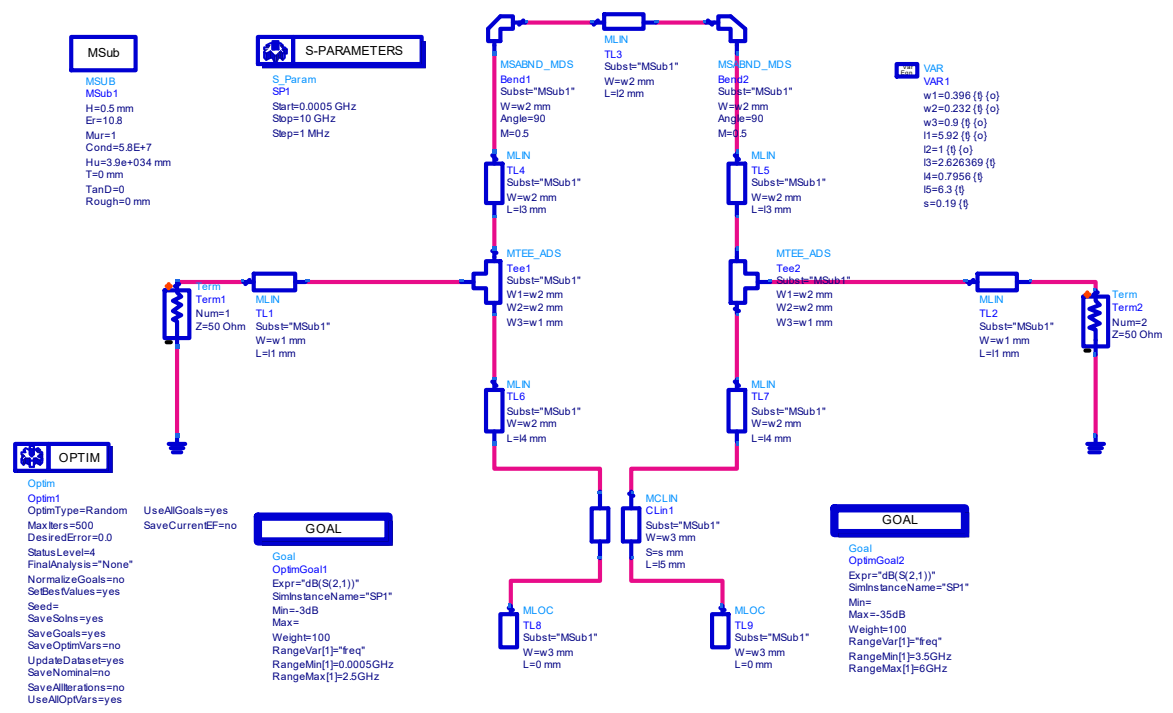

(a)

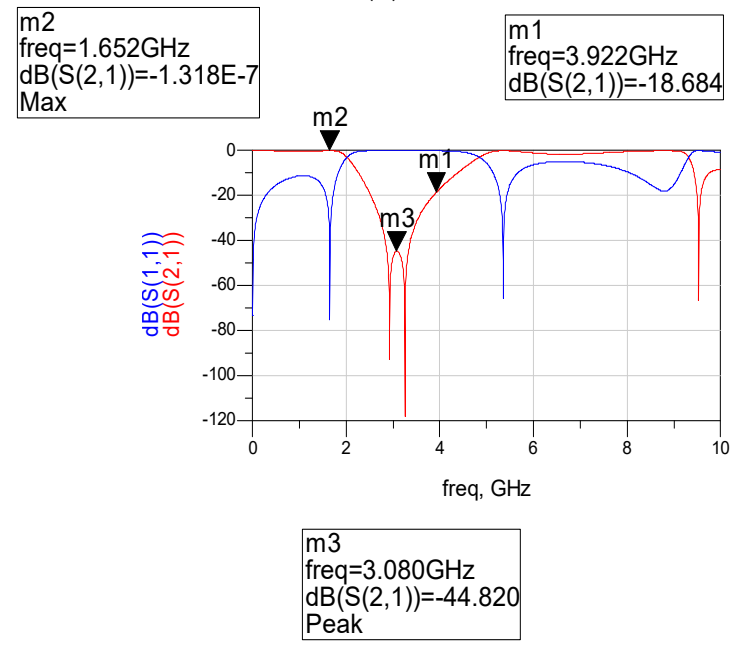

(b)

Figure 6. SIR hairpin LPF composed of single resonator with internal coupling. (a) The schematic diagram modeled in Agilent ADS software. (b) The simulation transmission characteristics.

As shown in Figure 6b, the proposed single SIR filter possesses the characteristic of high selectivity and produces two attenuation poles within a finite frequency band. This is the transmission characteristic of the three-branch elliptic function LPF. The disadvantage of this filter is that the SB width is narrow. Although the SB width can be properly enlarged by changing the size of each part of the SIR, it sacrifices the original characteristics of high frequency selectivity. Next, the SB width will be extended without changing the above structure sizes. That is to say, without changing the high edge frequency selectivity of the filter, the SB bandwidth is extended. The method is to introduce DGS structure.

\section{Model Simulation and Sample Measurement}

The proposed filter model is shown in Figure 7 using the design method of combining DGS with an SIR hairpin filter. The optimized dimensions of the SIR hairpin filter on the top layer are identical to those in Figure 5a. The sizes of the dielectric substrate and the three DGS patterns are the same with those in Figure 1c except that the length of the middle GDS $B_{2}$ is slightly longer than those of the two side GDS $B_{1}$. The optimized lengths of $B_{1}$ and $B_{2}$ are different in order to widen the valid SB 
width. The electrical parameters of the dielectric substrate and the size of each part of the filter are listed in Table 1.

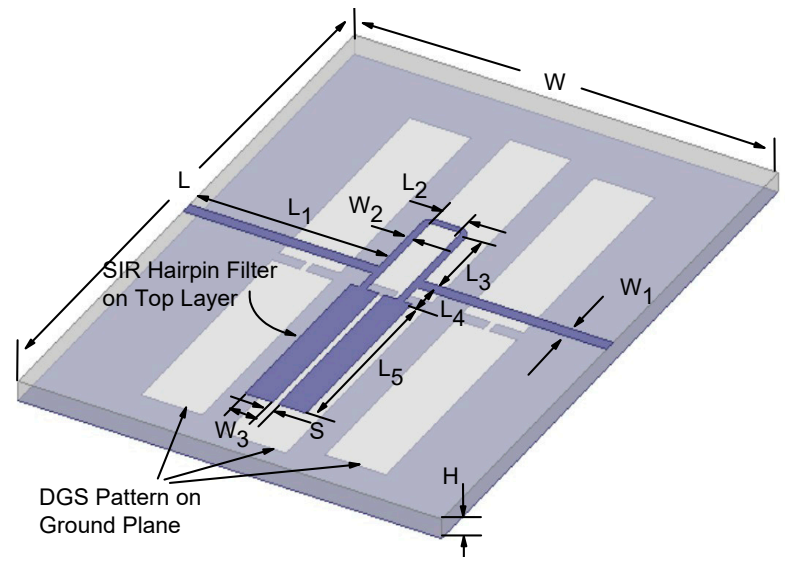

(a)

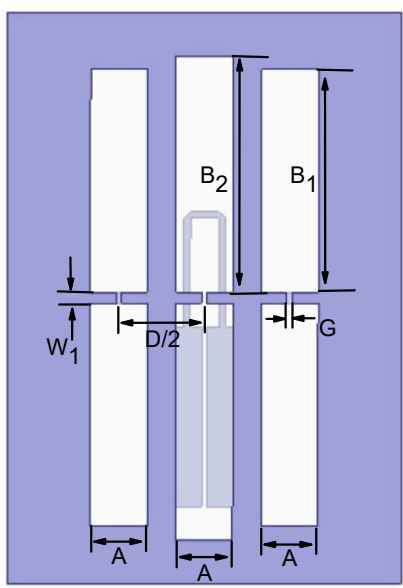

(b)

Figure 7. LPF model based on SIR and DGS. (a) 3-D model diagram and (b) DGS pattern diagram on the backside of the proposed filter.

The proposed filter is fabricated and shown in Figure 8. The Keysight N5224A PNA network analyzer is used to measure the S-parameters of the proposed LPFs. Figure 9 presents the simulated and measured results. The red lines are the S-parameters for a single SIR hairpin filter. These results obtained by full-wave electromagnetic simulation coincide with the results achieved by the circuit simulation using ADS software in Figure 6.

From the simulation results in Figure 9, it can be seen that the transmission coefficient of $S_{21, d B}$ is less than $0.38 \mathrm{~dB}$ from $0 \mathrm{~Hz}$ to $2 \mathrm{GHz}$, and the $\mathrm{SB}$ width is from $2.42 \mathrm{GHz}$ to $8.2 \mathrm{GHz}$ (see the blue curve) with a rejection of greater than $20 \mathrm{~dB}$. Fortunately, the testing results show a broader SB width from $2.42 \mathrm{GHz}$ to $9 \mathrm{GHz}$ (see the black curve) with the same frequency selectivity and the attenuation in SB. The upper SB value even exceeds $10 \mathrm{GHz}$ if the maximum attenuation degree is determined as $14.45 \mathrm{~dB}$. The simulation and testing results are in good agreement.

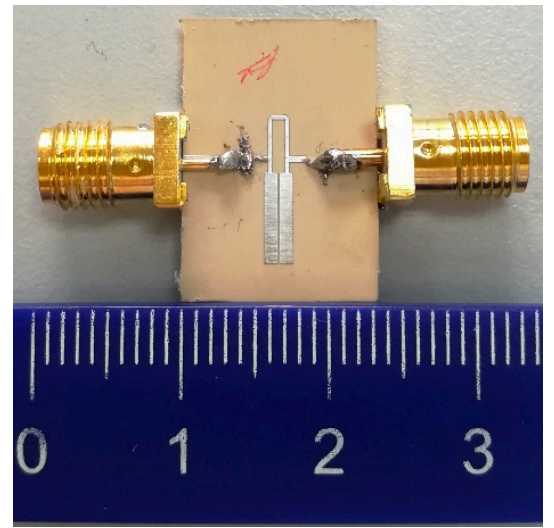

(a)

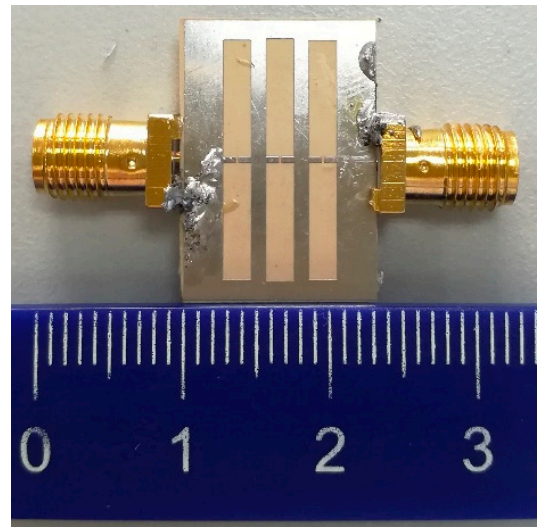

(b)

Figure 8. The fabricated microstrip LPF based on SIR and DGS: (a) top layer and (b) bottom layer. 


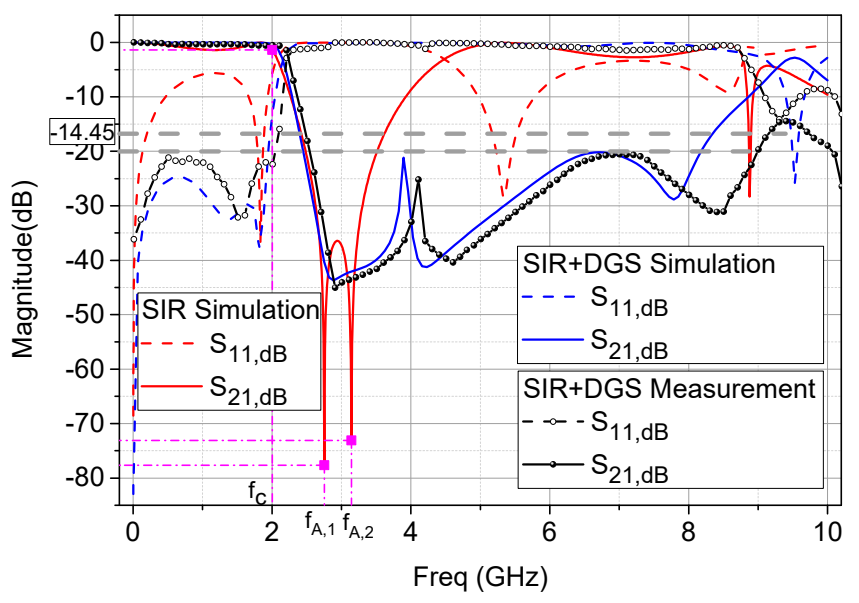

Figure 9. The simulation and measured results of S-parameters.

For the hairpin resonant filter, the simulated S-parameters shown in Figure 9 by the red curves can be used to obtain the input impedance $Z_{I N}[1]$ :

$$
Z_{I N}=\frac{1+\left(S_{11}+\frac{S_{12} S_{21} \Gamma_{L}}{1-S_{22} \Gamma_{L}}\right)}{1-\left(S_{11}+\frac{S_{12} S_{21} \Gamma_{L}}{1-S_{22} \Gamma_{L}}\right)} Z_{0}
$$

where $\Gamma_{L}=\left(Z_{L}-Z_{0}\right) /\left(Z_{L}+Z_{0}\right)$ is the load reflection coefficient, and $Z_{0}$ and $Z_{L}$ are the characteristic impedance of the transfer line and the load impedance, respectively. The $Z_{0}$ and $Z_{L}$ in our manuscript are also set to $50 \Omega$. Therefore, the $Z_{I N}=50\left(1+S_{11}\right) /\left(1-S_{11}\right)$ and its values are shown in Figure 10. From the $Z_{I N}$ in Figure $10 \mathrm{~b}$, it can be found that the first resonant frequency $f_{R, 1}=1 \mathrm{GHz}$. Therefore, the SIR hairpin filter is a resonant element.

The quality-factor $(Q)$ of a filter is the parameter to present the ratio of reactive power to active power. In the bandpass filter, the higher $Q$ values mean the narrower bandwidth of the filter [13]. Based on $Z_{I N}$, the quality-factor $(Q)$ value is given by $Q=\operatorname{Im}\left(Z_{I N}\right) / \operatorname{Re}\left(Z_{I N}\right)$. The $Q$ values reach 1120,1214 and 0.86 at the two attenuation poles $f_{A, 1}=2.75 \mathrm{GHz}, f_{A, 2}=3.14 \mathrm{GHz}$ and the cut-off frequency $f_{C}=2 \mathrm{GHz}$, respectively. The $\mathrm{Q}$ values theoretically would be 0 at the resonant frequency points. The $Q$ values at resonant frequency points $f_{R, 1}, \cdots, f_{R, 6}$ are very small values rather than zero, e.g., $Q_{f_{R, 1}}=0.0078$ at $f_{R, 1}$. This is because the very accurate frequency points for resonance failed to take using the discrete digital computing method.

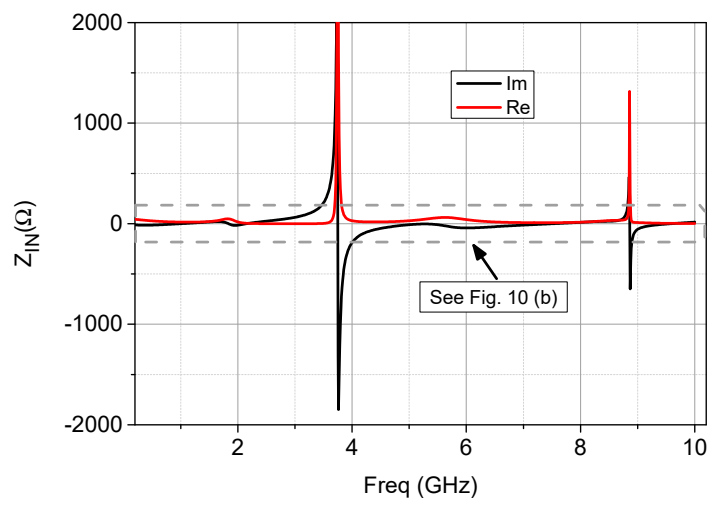

(a)

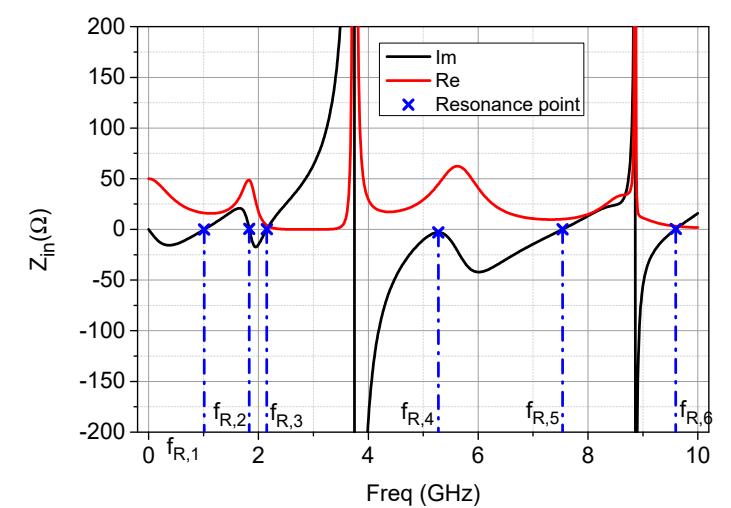

(b)

Figure 10. The input impedance of the SIR hairpin resonant filter. (a) Global view and (b) Partial view. 
According to the S-parameters calculated in Figure 9, we present the insertion loss (IL) in decibel form based on the equation of $I L_{d B}=10 \lg \left|S_{21}\right|^{2}=2 S_{21, d B}[1,14,15]$. Therefore, the values of $I L_{d B}$ are twice the values of $S_{21, d B}$ over the range of the investigated frequency.

The group delay as the derivative of the phase varies as an important parameter in microwave filter design. The smaller the group delay variation, the better the flat property of the designed filter. The simulation and measured values of group delay are shown in Figure 11. From this figure, the group delay varies from 0.23 to $0.78 \mathrm{~ns}$ in the pass band of $0 \sim 2 \mathrm{GHz}$. That is to say, the maximum variation of group delay is about $0.55 \mathrm{~ns}$, representing a good flat property of the designed filter.

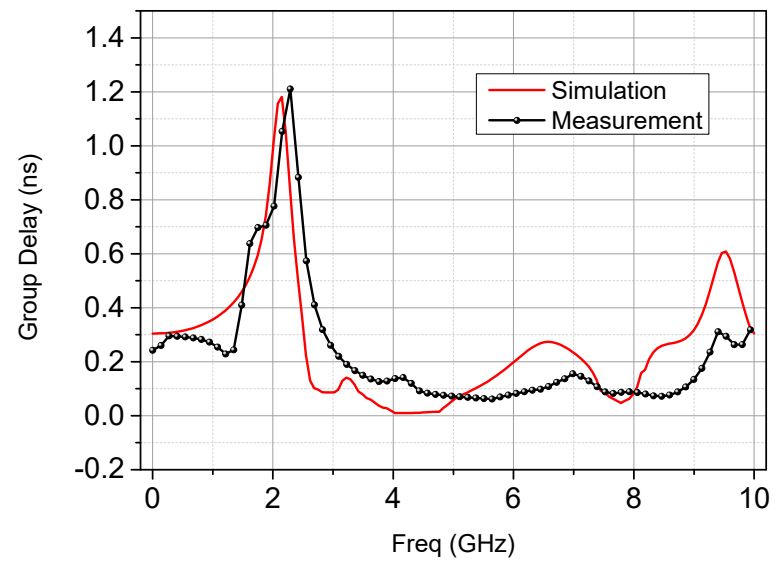

Figure 11. The simulation and measured values of group delay of the designed filter.

Some LPFs proposed in the references are compared with this paper in Table 2. The proposed LPF in this paper has smaller pass-band ripple (PBR) by comparing with the LPF in Reference [10]. The size of our designed LPF is less than those in Reference $[9,11]$ with similar SB and TB width. The attenuation in stop-band (ASB) of LPF in Reference [2] is better than that of our design, however, the size of the designed filter in the reference is larger than our proposed filter in this paper. Therefore, the LPF proposed in this paper possesses the properties of miniaturization, wide SB, high selectivity, and low PBR simultaneously.

Table 2. Performance comparisons between the reported filters and the proposed filter in this paper (SB: stop-band, TB: transition band, PBR: pass-band ripple, ASB: attenuation in stop-band).

\begin{tabular}{cccccc}
\hline Ref. & Size $/ \lambda$ & SB $(\mathrm{GHz})$ & TB $(\mathrm{GHz})$ & PBR (dB) & Substrate \\
\hline This work & $0.13 \times 0.09$ & $6.6^{*} ;>10^{* *}$ & $0.4^{*} ; 0.35^{* *}$ & $\leq 0.5$ & PTPE composite medium \\
{$[2]$} & $0.25 \times 0.08$ & $7.6^{* * *}$ & $0.4^{* * *}$ & low & RT / Duroid 6010 \\
{$[11]$} & $0.42 \times$ unavailable & $6.5^{*}$ & $0.5^{*}$ & low & ARLON $25 \mathrm{~N}$ \\
{$[9]$} & $0.13 \times 0.23$ & $6.7^{*}$ & $0.3^{*}$ & low & FR4 \\
{$[10]$} & $0.1 \times 0.1$ & $7.4^{*}$ & $0.2^{*}$ & high & FR4 \\
\hline
\end{tabular}

* ASB $<20 \mathrm{~dB} ;{ }^{* *} \mathrm{ASB}<14 \mathrm{~dB} ;{ }^{* * *} \mathrm{ASB}<30 \mathrm{~dB}$.

\section{Conclusions}

Firstly, the square-headed dumbbell-shaped DGS is introduced. The transmission characteristics of one-, two- and three-DGS units are analyzed and compared by the 3D numerical simulation software HFSS. The results show that the structure of DGS has the property of low-pass filtering, and the SB attenuation extremum frequency decreases with the increase of the area and number of DGS units.

Secondly, a compact LPF is designed by using SIR hairpin resonator with internal coupling, which is equivalent to three-branch elliptic function LPF. The optimal sizes of the SIR LPF are obtained by the engineering electromagnetic software ADS of Agilent company. From the result of the simulation, 
this compact hairpin LPF has high frequency selectivity and very low PB attenuation, but the SB width under $20 \mathrm{~dB}$ is not wide enough.

Finally, to extend the SB rejection width, we combine the DGS with the SIR LPF. By adjusting the area and number of DGS units, a microstrip LPF with small size, high frequency selectivity and wide SB suppression is achieved. Meanwhile, the maximum group delay variation in the passband is within $0.55 \mathrm{~ns}$. The manufactured compact filter keeps good consistency with the simulation results, which proves the validity of the theoretical design. This compact LPF with excellent transmission performance is very suitable for the application of modern wireless communication circuits.

Author Contributions: Conceptualization, J.Z.; Methodology, J.Z.; Software, J.Z.; Validation, J.Z.; Formal analysis, J.Z.; Investigation, C.Z.; Resources, J.Z.; Data curation, J.Z.; Writing—original draft preparation, R.-S.Y. and J.Z.; Writing-review and editing, J.Z.

Funding: This research was supported in part by the open research fund of the National and Local Joint Engineering Laboratory of RF Integration and Micro-Assembly Technology (KFJJ20180203), the Scientific Research Foundation for the High-Level Talents of Jinling Institute of Technology (jit-b-201719), and the Scientific Research Incubation Foundation of Jinling Institute of Technology (jit-fhxm-201802).

Acknowledgments: The authors would like to thank D. Chen for experimental support to this work.

Conflicts of Interest: The authors declare no conflict of interest.

\section{References}

1. Pozar, D.M. Microwave Engineering, 2nd ed.; Wiley: New York, NY, USA, 1998; Chapter 8.

2. Lung-Hwa, H.; Kai, C. Compact elliptic-function low-pass filters using microstrip stepped-impedance hairpin resonators. IEEE Trans. Microw. Theory Tech. 2003, 51, 193-199. [CrossRef]

3. Liu, X.; Wu, W.; Ji, P.; Yuan, N. Design of Compact Dual-Passband Filters by Parasitic Passband With Controllable Passbands. IEEE Microw. Wirel. Compon. Lett. 2018, 28, 410-412. [CrossRef]

4. Saal, R.; Ulbrich, E. On the Design of Filters by Synthesis. IRE Trans. Circuit Theory 1958, 5, $284-327$. [CrossRef]

5. Sheen, J. A compact semi-lumped low-pass filter for harmonics and spurious suppression. IEEE Microw. Guided Wave Lett. 2000, 10, 92-93. [CrossRef]

6. Mora, S.; Alonso, Y.; Vargas, N.; Vera, J.; Avendano, J. Design of a bandpass filter using microstrip Hairpin resonators. In Proceedings of the 2017 CHILEAN Conference on Electrical, Electronics Engineering, Information and Communication Technologies (CHILECON), Pucon, Chile, 18-20 October 2017; pp. 1-5.

7. Ahn, D.; Park, J.; Kim, C.; Kim, J.; Qian, Y.; Itoh, T. A design of the low-pass filter using the novel microstrip defected ground structure. IEEE Trans. Microw. Theory Tech. 2001, 49, 86-93. [CrossRef]

8. Nechel, E.V.; Ferranti, F.; Latairc, J.; Rolain, Y. Efficient Design Optimization and Variability Analysis of Defected Ground Structure Filters Using Metamodels. In Proceedings of the 2017 IEEE MTT-S International Microwave and RF Conference (IMaRC), Ahmedabad, India, 11-13 December 2017; pp. 1-4.

9. Cao, S.; Han, Y.; Chen, H.; Li, J. An Ultra-Wide Stop-Band LPF Using Asymmetric Pi-Shaped Koch Fractal DGS. IEEE Access 2017, 5, 27126-27131. [CrossRef]

10. Zeng, Z.; Yao, Y.; Zhuang, Y. A Wideband Common-Mode Suppression Filter with Compact-Defected Ground Structure Pattern. IEEE Trans. Electromagn. Compat. 2015, 57, 1277-1280. [CrossRef]

11. Kufa, M.; Raida, Z. Lowpass filter with reduced fractal defected ground structure. Electron. Lett. 2013, 49, 199-201. [CrossRef]

12. Makimoto, M.; Yamashita, S. Microwave Resonators and Filters for Wireless Communication Theory, Design and Application; Springer: Berlin, Germany, 2001; Chapter 4.

13. Ahmadivand, A.; Sinha, R.; Gerislioglu, B.; Karabiyik, M.; Pala, N.; Shur, M. Transition from capacitive coupling to direct charge transfer in asymmetric terahertz plasmonic assemblies. Opt. Lett. 2016, 41, 5333-5336. [CrossRef] [PubMed] 
14. Gerislioglu, B.; Ahmadivand, A.; Pala, N. Tunable plasmonic toroidal terahertz metamodulator. Phys. Rev. B 2018, 97, 161405. [CrossRef]

15. Kruger, B.A.; Joushaghani, A.; Poon, J.K.S. Design of electrically driven hybrid vanadium dioxide $\left(\mathrm{VO}_{2}\right)$ plasmonic switches. Opt. Express 2012, 20, 23598. [CrossRef] [PubMed] 